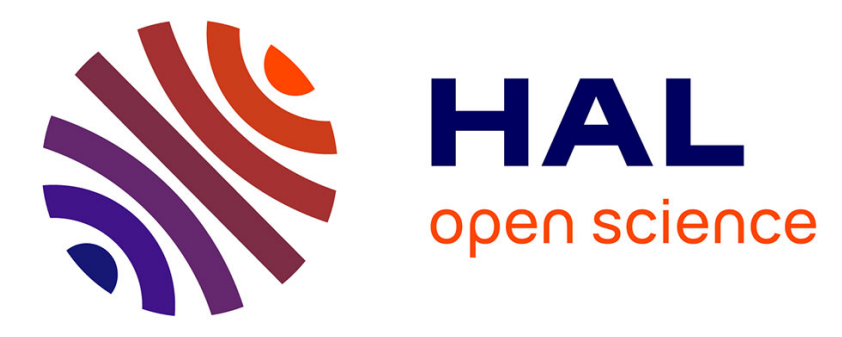

\title{
Manipulation of the fluorescence of nanocrystals by opal-based heterostructures
}

Bourdillon Céline, Hong Phan Ngoc, Daney de Marcillac Willy, Coolen Laurent, Agnès Maître, Catherine Schwob

\section{- To cite this version:}

Bourdillon Céline, Hong Phan Ngoc, Daney de Marcillac Willy, Coolen Laurent, Agnès Maître, et al.. Manipulation of the fluorescence of nanocrystals by opal-based heterostructures. Journal of Materials Chemistry C, 2015, 3 (37), pp.9734-9739 10.1039/C5TC01829C . hal-01203465

\section{HAL Id: hal-01203465 https: / hal.sorbonne-universite.fr/hal-01203465}

Submitted on 23 Sep 2015

HAL is a multi-disciplinary open access archive for the deposit and dissemination of scientific research documents, whether they are published or not. The documents may come from teaching and research institutions in France or abroad, or from public or private research centers.
L'archive ouverte pluridisciplinaire $\mathbf{H A L}$, est destinée au dépôt et à la diffusion de documents scientifiques de niveau recherche, publiés ou non, émanant des établissements d'enseignement et de recherche français ou étrangers, des laboratoires publics ou privés. 


\title{
Manipulation of the fluorescence of nanocrystals by opal-based heterostructures
}

\author{
Bourdillon Céline $^{\mathrm{a}, \mathrm{b}}$, Hong Phan Ngoc ${ }^{\mathrm{c}}$, Daney de Marcillac Willy ${ }^{\mathrm{a}, \mathrm{b}}$, Coolen Laurent ${ }^{\mathrm{a}, \mathrm{b}}$, Maître \\ Agnès $^{\mathrm{a}, \mathrm{b}}$, Schwob Catherine $\mathrm{e}^{\mathrm{a}, \mathrm{b}}$.
}

\author{
5 Received (in $X X X, X X X) X$ th $X X X X X X X X X 20 X X$, Accepted $X$ th $X X X X X X X X X 20 X X$ \\ DOI: 10.1039/b000000x
}

Opal-based photonic crystals are promising materials to engineer complex heterostructures for efficient manipulation of nano-emitters fluorescence. We fabricated and characterized a structure composed of a layer of silica embedded between two silica opals. Thanks to this 10 controlled planar defect which opens a permitted frequency band in the photonic stopband, an increase of emission, depending on angular distribution, is evidenced through photoluminescence spectroscopy. We discuss the use of such a structure as a self-assembled micrometer-sized spectroscopic device and demonstrate that it can be used to point the maximum emission wavelength of an unknown light source up to a certain linewidth. It can as

15 well separate two sources, emitting at different wavelengths, with a resolution given by the Rayleigh criterion.

\section{Introduction}

Because they offer particular optical properties, such as photonic bandgaps, photonic crystals are intensively ${ }_{20}$ developed and studied. ${ }^{1}$ They are defined by a periodicity of the optical index at a scale comparable to the optical wavelength, in 1, 2 or 3 dimensions $^{1}$, and can be fabricated by various techniques. Lithographic techniques are the most used to engineer 1D- and 2D-photonic crystals. They can ${ }_{25}$ also provide complex and versatile $3 \mathrm{D}$-photonic crystals ${ }^{2-5}$ but at the cost of heavy and long processes, leading to small size samples. An alternative consists in processes based on the self-assembly of colloidal nanospheres solutions such as sedimentation $^{3,4}$, Langmuir ${ }^{5,6}$ or convective methods ${ }^{7,8}$. The

30 resulting 3D-photonic crystals composed of compact stackings of silica or polymer spheres, also called opals, present a well-defined face-centered cubic crystalline structure over centimetre scales.

The periodicity of an opal can be disrupted by the 35 introduction of a controlled defect in the structure, which opens a permitted band in the forbidden photonic stopband. Various techniques ${ }^{9}$ have been developed to engineer defects in opals. Among them, the integration of a planar defect, made by Langmuir methods ${ }^{5,6,10}$ or material sputtering ${ }^{11}$, has 40 been successfully realized.

Photonic crystals, and particularly opals, are of great interest to modify the emission properties of fluorescent nanoparticles such as organic molecules ${ }^{12-14}$ or quantum $\operatorname{dots}^{13,15,16}$. Indeed, when the emission wavelength of the 45 emitter corresponds to the stopband, the emission intensity can be strongly decreased ${ }^{17,18}$ and the fluorescence lifetime can increase ${ }^{17-20}$. In literature, opals were also used in plasmonic devices to manipulate light properties: as templates to fabricate a plasmonic grating ${ }^{21,22}$, as hybrid ${ }_{50}$ photonic-plasmonic crystals $^{23}$ or coupled to metallic quantum dots ${ }^{24}$. Moreover, the modification of the opal reflexion properties due to pressure, temperature, chemical or biological stimuli can be used to create a sensor, and integration of nanocrystals in opals has been suggested in
55 order to create active sensor materials. ${ }^{25}$

In this paper, we present an efficient method to angularly filter the fluorescence of colloidal nanocrystals with an opalbased heterostructure, composed of a layer of sputtered silica between two silica opals. We demonstrate, by measurements 60 of emission diagrams, that the photonic crystal affects the detected fluorescence of nanocrystals. The use of such a structure for the spectral determination of one or two unknown light sources is discussed. Its ability to be used as a filter device is evaluated in terms of resolution.

\section{${ }_{65}$ Synthesis and characterization of the opal- based heterostructure}

The hererostructure of interest is composed of a planar defect sandwiched between two silica opals. The opals are fabricated by self-assembly from home-made silica spheres. 70 The spheres are synthetized using a procedure derived from the Stöber-Fink-Bohn method ${ }^{26}$ : as published previously ${ }^{27}$, we obtain spherical and uniform particles with a mean diameter of $343 \mathrm{~nm}$ and a size dispersion of $5.7 \%$. The selfassembly technique used to prepare the opals from these 75 spheres is the convective method. ${ }^{7}$ In this method, a substrate, tilted with an angle of $10^{\circ}$ with respect to the vertical direction, is placed in a vessel containing a colloidal solution of silica spheres at a concentration of $2 \% \mathrm{w} / \mathrm{v}$ in ethanol. A temperature gradient, applied between the bottom 80 of the vessel and the surface of the solution, facilitates the evaporation of the solvent, leading to a convective motion which brings the spheres to the meniscus where they selforganize. With the chosen parameters, we obtain wellordered crystallographic structures, as demonstrated by the 85 different crystallographic planes visible on Figure 1. The opal thickness is of the order of 12 layers $(4.1 \mu \mathrm{m})$. 


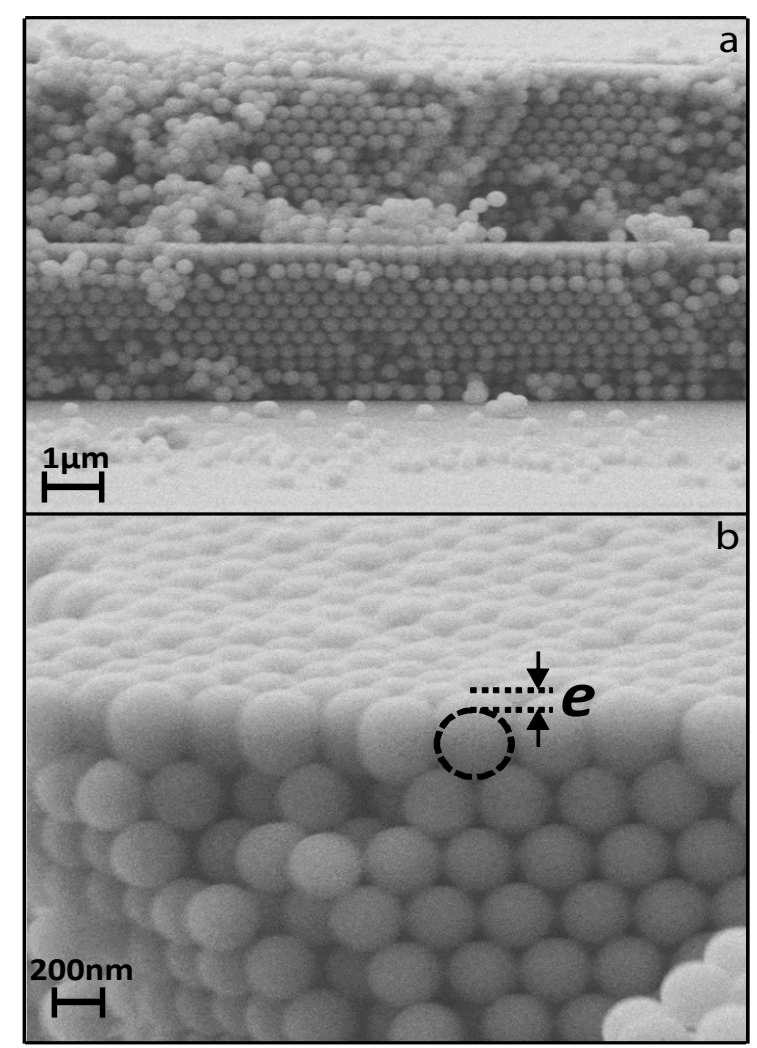

Figure 1. SEM images of a cross section a- of the opal-silica layeropal heterostructure, b- with a zoom on the defect mode before the deposition of the upper opal.

5 The planar defect is obtained by deposition of a given thickness of silica on the surface of an opa ${ }^{11}$. Silica (with optical index equal 1.49) was sputtered from a $\mathrm{SiO}_{2}$ target under argon atmosphere. The opal surface was oriented to be parallel to the target so that the sputtering took place with a 10 preferred direction, perpendicularly to the surface. The parameters which influence the crystallinity and the thickness of the sputtered layer are the nature of the gas, the resulting pressure in the deposition chamber, the temperature of the substrate, the distance between the silica target and the 15 substrate (which has to be slightly larger than the mean free path of the silica particles) and the duration of the process. We worked with a residual pressure of argon of $10^{-3} \mathrm{mbar}$, which corresponded to a mean free path between 5 and $6 \mathrm{~cm}$. Consequently, the distance between the target and the 20 substrate was set to $6 \mathrm{~cm}$. The temperature of the substrate was on the order of $100^{\circ} \mathrm{C}(0.07$ times the fusion temperature of silica). Under these sputtering conditions, the deposited silica forms columns on top of the spheres of the opal upper layer, leading to a structure that reproduces the opal ${ }_{25}$ periodicity (fig. 1b). To complete the heterostructure, the sputtered silica layer is covered by a second opal similar to the first one. As it induces a disruption of the opal periodicity in the thickness direction, the planar defect creates a frequency passband inside the opal photonic bandgap,
30 analog to the role of defects in crystalline solids. ${ }^{28}$ In other words, the silica layer constitutes an optical Fabry Perot-like cavity, with the opals in their forbidden band acting as mirrors. At the output of the cavity, maxima of transmitted light are obtained for the corresponding wavelengths. The 35 spectral position of the passband depends on the thickness of sputtered silica (see simulations in the supporting information part). For the sample studied here, the thickness $e$ of the defect layer, determined from SEM images as the height of the elongated beads minus the opal spheres 40 diameter (fig. 1b), is $112 \mathrm{~nm}$.

The heterostructure was optically characterized by angleresolved specular reflection spectroscopy. ${ }^{29}$

The spectra were recorded, from the air-opal interface, at various incidence angles $\theta$, defined from the normal to the ${ }_{45}$ (111) crystallographic planes (sample surface). The incident beam was provided by a supercontinuum source connected to an optical fiber, mounted on a goniometer arm with a collimator to obtain a light spot of $2 \mathrm{~mm}$ on the sample. The reflected beam was collected by a symmetric collimated 50 fiber. The detected signal was analysed by a spectrometer (resolution $\Delta \lambda=1.5 \mathrm{~nm}$ ). All spectra were normalized by the source spectrum, measured with the two fibers facing each other. As the incident light was unpolarized, the detected signal included both s- and p-polarizations.

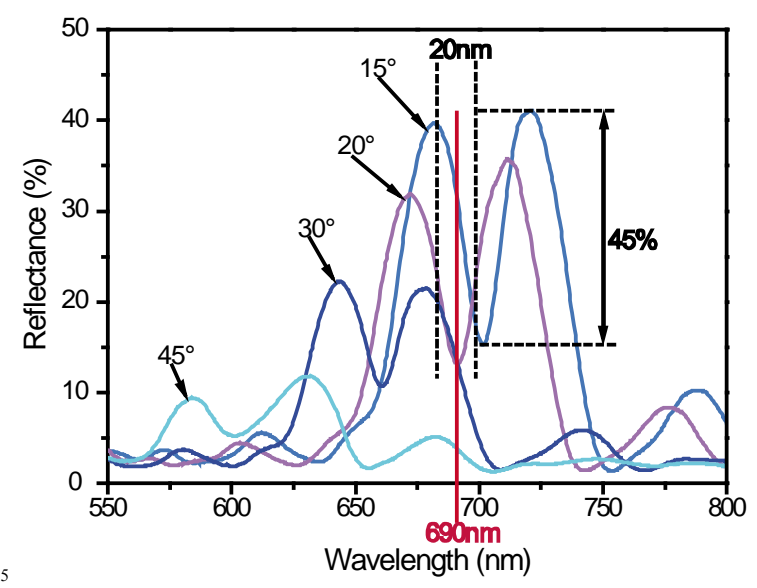

Figure 2. Specular reflection spectra of the heterostructure at different angles $\theta$.

Figure 2 shows the reflection spectra for specular angles $\theta$ ranging from $15^{\circ}$ to $45^{\circ}$. For each angle the signal displays a 60 sharp dip of $20 \mathrm{~nm}$ Full Width at Half Maximum (FWHM) (shown with dotted lines for the $20^{\circ}$-spectrum on Figure 2) corresponding to the defect mode caused by the planar defect (passband and so increase of the transmitted light), within a broader peak which is the signature of the opal stopband. ${ }_{65}$ Indeed, if a wavelength of the white source spectra is located in the stopband, the corresponding light cannot propagate through the photonic crystal and is consequently reflected. As opals present incomplete bandgaps (light propagation is not forbidden for all directions), the reflected wavelengths 70 depend on the angle and follow approximatively Bragg's law. The defect mode dip is shifted to shorter wavelengths, when the angle $\theta$ increases, as expected in a Fabry Perot-like cavity. The thickness of the sputtered silica layer was chosen 
so that the defect mode was located in the middle of the stopband. To quantify the depth of the defect mode, we defined the figure of merit $\mathrm{F}$ of the heterostructure as $\mathrm{F}=$ $\left(R_{\max }-R_{\min }\right) /\left(R_{\max }+R_{\min }\right), R_{\max }$ and $R_{\min }$ being respectively $s$ the reflection maximum of the spectrum and the minimum of reflection corresponding to the defect mode. The high measured value of $\mathrm{F}, \mathrm{F} \sim 45 \%$ is an indication of the heterostructure good quality and in particular of the epitaxial growth of the second opal on top of the first one. ${ }^{11}$

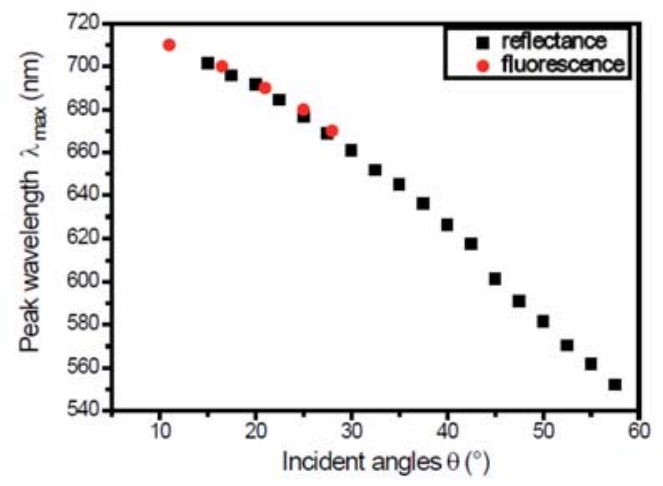

Figure 3. Plot of the wavelengths of the defect mode dips in reflection measurements (black squares) and peaks in fluorescence measurements (red points) as a function of the specular or collection angle respectively.

15 On Figure 3, we plot the central wavelength of the defect mode for both reflectance and fluorescence (presented in the next part) measurements as a function of the angle $\theta$. By varying $\theta$ from $15^{\circ}$ to $57.5^{\circ}$, this one can be tuned over more than $150 \mathrm{~nm}$. We establish here a clear relationship between

20 the central wavelength of the passband and the angle $\theta$. We will use it, in the last part of this article, to convert the angles obtained by emission diagrams measurements into wavelengths.

\section{${ }_{25}$ Angle-resolved photoluminescence spectro- goniometry}

The opal-based heterostructure was used to manipulate the fluorescence of colloidal semi-conductor $\mathrm{CdTeSe} / \mathrm{ZnS}$ coreshell nanocrystals with a fluorescence maximum at $705 \mathrm{~nm}$ 30 and a full width at half maximum of $60 \mathrm{~nm}$. Its effects on emission were evidenced by photoluminescence spectrogoniometry.

The experimental setup is presented on Figure 4. A $3 \mu 1$ dropplet of $1 \mu \mathrm{M}$ solution of nanocrystals in decane was 35 spread on a glass slide. After solvent evaporation, the heterostructure described above was placed on the slide. The nano-emitters were pumped by a cw blue laser diode (wavelength $473 \mathrm{~nm}$, power $100 \mathrm{~mW}$ ) at fixed incidence. The fluorescence transmitted through the heterostructure 40 was collected by an optical fiber mounted on a rotation stage to change the angle of detection $\theta$ (with respect to vertical direction normal to the sample) ${ }^{18}$. The distance between the sample and the fiber was around $10 \mathrm{~cm}$, the size of the illumination spot, $25 \mathrm{~mm}^{2}$, and the angular resolution, 45 approximately $3^{\circ}$. The signal was analysed with a spectrometer (Jobin Yvon HR460) coupled to a nitrogen- cooled Si-CCD camera. The spectral resolution of the setup was $1 \mathrm{~nm}$.
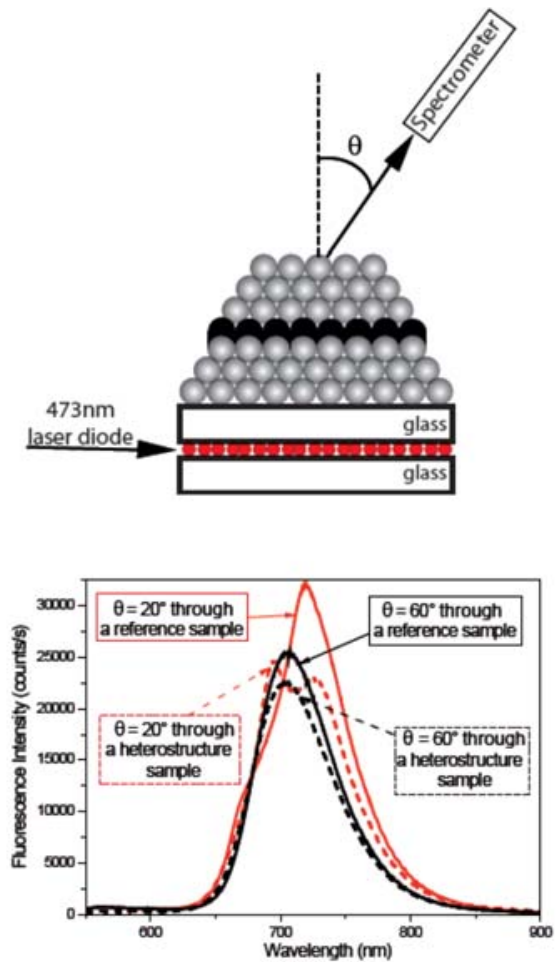

Figure 4. Experimental setup (top) and emission spectra (down) of the nanocrystals through an opal with defect mode (named heterostructure sample) and an opal without defect mode (named 55 reference sample) for $20^{\circ}$ and $60^{\circ}$ angles.

Figure 4 shows the photoluminescence spectra recorded at various $\theta$ angles through the heterostructure sample (planar defect between two opals) and through a single opal without planar defect, used as a reference sample. The spectra were 60 centered at $705 \mathrm{~nm}$ and their differences in intensity and shape originated from the stopband and passband (defect mode) effects which spatially and spectrally filter the emission.

A clearer representation consists in plotting the emission ${ }_{65}$ diagram, the collected intensity as a function of the angle for a given emission wavelength. Figure 5(a) shows the emission diagram at $690 \mathrm{~nm}$ for $\theta$ ranging from $0^{\circ}$ to $90^{\circ}$ for the heterostructure sample and the reference sample. 


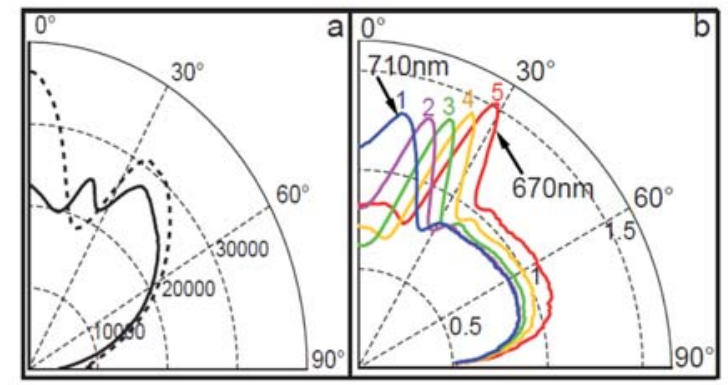

Figure 5. a- Angular emission of the nanocrystals through the heterostructure sample (plain line) and the reference sample (dotted line) at $690 \mathrm{~nm}$. b- Angular emission of the nanocrystals 5 through the heterostructure sample normalized by the emission through the reference sample at different wavelengths (1: 710 $\mathrm{nm}, 2: 700 \mathrm{~nm}$, 3: $690 \mathrm{~nm}, 4: 680 \mathrm{~nm}, 5: 670 \mathrm{~nm}$ ).

At large angles $\left(90^{\circ}\right)$, for both samples, the collected fluorescence was very low and increased as the angle 10 decreased due to the Lambertian nature of the emission diagram. For angles between $40^{\circ}$ and $0^{\circ}$, the emission diagram differed from a Lambertian one: a large dip in the fluorescence intensity, corresponding to the photonic bandgap, was observed. For the heterostructure sample, a 15 sharper peak (roughly $15^{\circ}$ of angular width) appeared in the dip evidencing the passband induced by the planar defect.

To highlight this fluorescence peak, we plot on Figure $5 \mathrm{~b}$ the emission through the heterostructure normalized by the emission through the reference sample at the same 20 wavelength, for different wavelengths in the stopband of the opal. The passband signature appeared clearly as a sharp peak. For high angles (larger than $40^{\circ}$ ), the ratio between the two signals was almost constant and close to one (it decreased above $80^{\circ}$ due to normalization between two 25 vanishing signals). A slight dip can be observed on both sides of the peak. It comes from the fact that the stopband width is larger in the case of the heterostructure (as it can be observed on Figure 5a): this leads to a normalization with an artificially high value of the signal, and so to the appearance 30 of a dip.

The angular positions of the maxima of fluorescence for different wavelengths (Figure 5b) are in very good agreement with the minima of reflection (Figure 2), as evidenced by the plot of the central wavelengths, for both

35 fluorescence peaks and reflection dips, as a function of the angle $\theta$, shown of Figure 3. This allows a direct conversion between angular and spectral positions from the experimental values.

We demonstrated that this opal-based heterostructure is an 40 interesting tool for an efficient spectrally-dependent angular filtering of the emission of nanocrystals.

\section{Light sources characterization}

In this part, we show, through the exploitation of the 45 experimental emission diagrams, that the opal-based heterostructure can provide an efficient micrometer-size spectroscopic device, leading to a spectral information on different kinds of light sources. Moreover, we demonstrate that this structure can be used to spatially separate two
50 sources, emitting at different wavelengths, and we evaluate the corresponding resolution.

Determination of the central wavelength of a light source

The photoluminescence experiments shown on Figure 5 55 were obtained with $60 \mathrm{~nm}$-spectral width nanocrystals and analysed with a $1 \mathrm{~nm}$ resolution, leading to emission diagrams recorded each nm. From these experimental results, we evidenced a clear relationship between the angle $\theta_{\mathrm{c}}$ corresponding to the maximum of the fluorescence peak ${ }_{60}$ and the wavelength $\lambda_{c}$ corresponding to the maximum of the reflection dip.

We want now to extract a spectral information from a broad light source by summing the recorded emission diagrams at different wavelengths. This is equivalent to measuring the 65 light transmitted by a broad source through the heterostructure in the far field on a simple CCD camera.

The photoluminescence measurements allow us to determine the emission diagram when the heterostructure is illuminated by a Gaussian source of variable spectral width $\delta \lambda$. Details 70 on the reconstructed fluorescence intensity are given in the supporting information part.

We plot on Figure 6 the reconstructed signal for different widths of the light source.

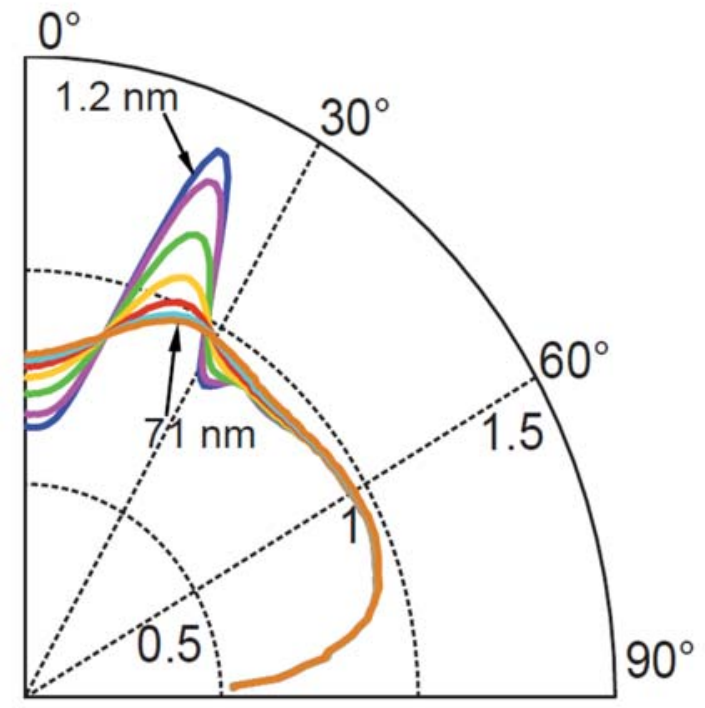

75 Figure 6. Reconstructed normalized angular emission for a source of gaussian spectrum centered at $690 \mathrm{~nm}$ and different $\delta \lambda=$ $1.2 \mathrm{~nm}, 12 \mathrm{~nm}, 24 \mathrm{~nm}, 36 \mathrm{~nm}, 48 \mathrm{~nm}, 60 \mathrm{~nm}, 71 \mathrm{~nm}$.

We can point the maximum of the fluorescence peak, and so deduce the spectral position of the maximum from the 80 reconstructed emission diagram. We measure a value of the angle $\theta_{c}$ of $21^{\circ}$ (with $\pm 3^{\circ}$ resolution, due to the photoluminescence experimental setup) and recover the expected value of the central wavelength of the light source $\lambda_{\mathrm{c}}=690 \mathrm{~nm}$ (with $\pm 15 \mathrm{~nm}$ resolution) from the relationship 85 established in Figure 3. This method can be used as long as the source width is not too broad compared to the defect mode width. Indeed it is applicable up to $60 \mathrm{~nm}-\mathrm{FWHM}$ light sources. 
Spectral resolution for a superposition of two light sources

5 In the previous part, we have shown that the emission diagrams of nanocrystals could be used to determine the central wavelength of a light source until a critical width. In this part, we consider the case of a mixture of two kinds of fluorophores, emitting at different wavelengths, and 10 demonstrate that they can be spectrally resolved thanks to their fluorescence diagrams recorded through the heterostructure. As previously, we consider, two sources with central wavelengths $\lambda_{1}$ and $\lambda_{2}$ and widths $\delta \lambda_{1}$ and $\delta \lambda_{2}$ respectively, separated by $\Delta \lambda\left(\Delta \lambda=\left|\lambda_{2}-\lambda_{1}\right|\right)$. By summing the 15 corresponding reconstructed emission diagrams, we obtained the angular response of the two mixed sources. On Figure 7, we plotted the emission diagrams for a source width $\delta \lambda=1.2 \mathrm{~nm}$ and different $\Delta \lambda$ decreasing from $40 \mathrm{~nm}$ (Figure 7a) to $20 \mathrm{~nm}$ (Figure 7c).

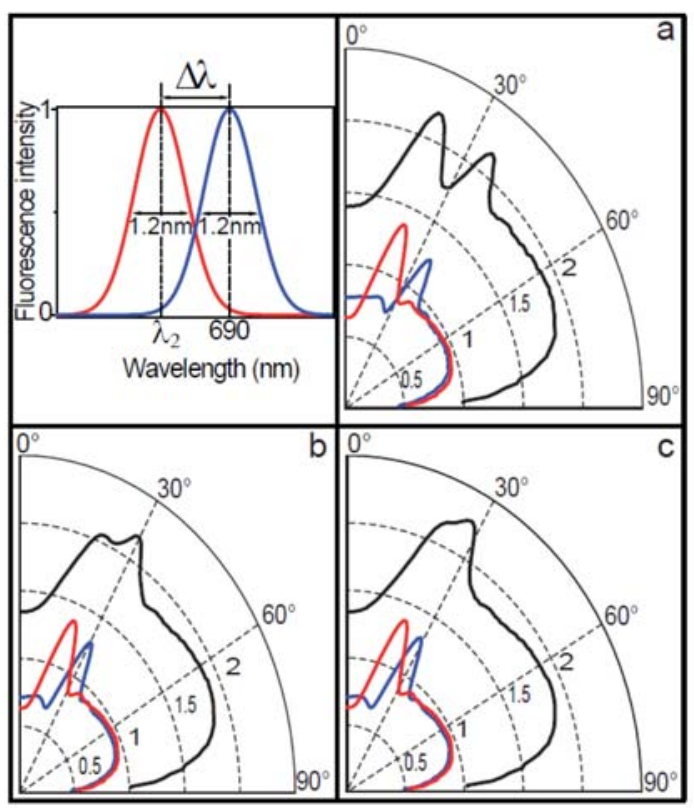

20

Figure 7. Reconstructed angular emission diagrams of the $1.2 \mathrm{~nm}$ width sources (red and blue lines) and the sum (black line) of the diagrams for different $\Delta \lambda$ : a- $\Delta \lambda=40 \mathrm{~nm} ;$ b- $\Delta \lambda=25 \mathrm{~nm}$; c- $\Delta \lambda=20 \mathrm{~nm}$

The two sources contributions can be distinguished on the 25 emission diagrams if the central wavelengths are separated by at least $25 \mathrm{~nm}$.

Finally, the sources widths were expanded to $12 \mathrm{~nm}, 24 \mathrm{~nm}$ and $36 \mathrm{~nm}$ to compare with the sharper ones. For each width, we plotted on Figure 8, the emission diagrams corresponding 30 to the resolution limit $\Delta \lambda_{\min }$.

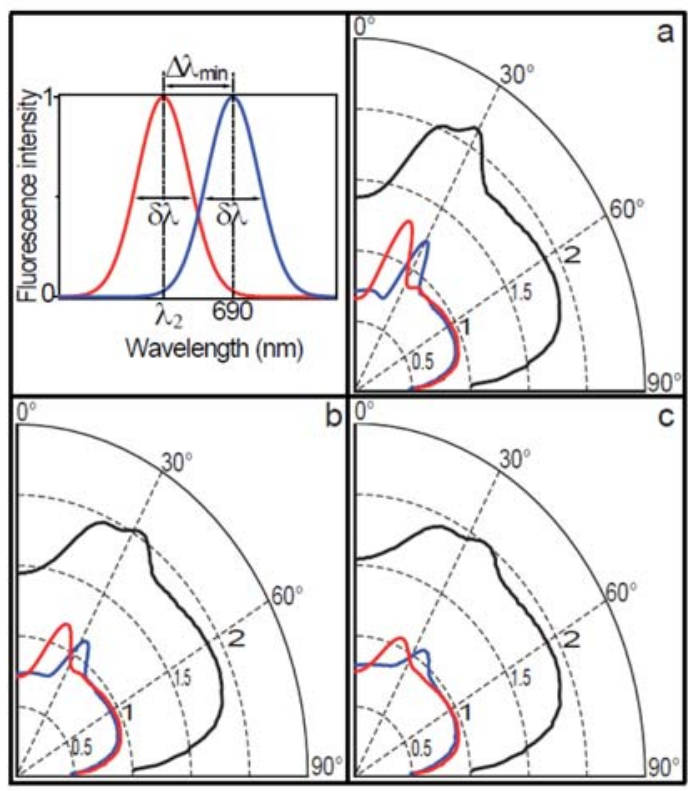

Figure 8. Reconstructed angular emission diagrams (red and blue lines) and the sum (black line) of the emission diagrams of two sources of different $\delta \lambda$ at the corresponding $\Delta \lambda_{\min }$ : a- $\delta \lambda=12 \mathrm{~nm}$, 45 $\Delta \lambda_{\min }=25 \mathrm{~nm} ; \mathrm{b}-\delta \lambda=24 \mathrm{~nm}, \Delta \lambda_{\min }=30 \mathrm{~nm} ; \mathrm{c}-\delta \lambda=36 \mathrm{~nm}, \Delta \lambda_{\min }=40 \mathrm{~nm}$.

For quasi-monochromatic sources $(\delta \lambda=1.2 \mathrm{~nm}$ and $\delta \lambda=12 \mathrm{~nm})$, sharper than the defect mode FWHM (20 nm, see Figure 2), the resolution is limited by the heterostructure and is equal to $\Delta \lambda_{\lim }=25 \mathrm{~nm}$. For broader sources $(\delta \lambda=24 \mathrm{~nm}$ and ${ }_{50} \delta \lambda=36 \mathrm{~nm}$ ), larger than the defect mode FWHM, the resolution is limited by the source and given by the Rayleigh criterion. Finally, we could distinguish sources of maximum widths equal to $36 \mathrm{~nm}$, separated by at least $40 \mathrm{~nm}$. In the case of two sources of different widths, the resolution limit 55 is given by the larger source.

\section{Conclusion}

Opal-based heterostructures are efficient tools to manipulate the fluorescence of nano-emitters. We demonstrated a 60 significant angular filtering of the emission through emission diagrams inferred from photoluminescence spectroscopy. From the experimental data, we proposed an original use of these structures, as a self-organized micrometer-sized monochromating device, to determine the central 65 wavelength of an unknown light source, from the knowledge of the angular position of the fluorescence peak. With this method, light sources as broad as $60 \mathrm{~nm}-\mathrm{FWHM}$ can be characterized. We also studied the case of two mixed unknown light sources and shown that they can be 70 distinguished until a $36 \mathrm{~nm}-\mathrm{FWHM}$ with a resolution limit 
of $40 \mathrm{~nm}$. Coupled to a simple camera, these structures can provide an efficient device for spectral characterization.

\section{Supporting information}

\section{Simulation of the heterostructure}

5 To achieve a complete study of the heterostructure, we simulated the $0^{\circ}$-transmission spectra (instead of reflection spectra for computing power reasons) of our samples illuminated by a light source of constant intensity over the visible range. Simulations have been performed with the 10 Finite Difference Time Domain (FDTD) method, using the freely available software MEEP developed in MIT. ${ }^{30}$ The computed structure is shown on the inset of Figure 9. For the lower and upper opals, the spheres diameter and silica index values, deduced from structural and optical 15 characterizations ${ }^{29}$, were set respectively to $\mathrm{D}=343 \mathrm{~nm}$ and $\mathrm{n}_{1}=1.32$. To reproduce the shape of the defect made of sputtered silica, a monolayer of spheres of same diameter D and of index $n_{2}=1.49$ was inserted between the two opals ( $n_{1}$ is lower than $\mathrm{n}_{2}$ because of the porosity of the silica spheres 20 synthetized by sol-gel method). The position of these spheres was set so that the distance between their top and the top of the first opal upper layer was equal to the thickness $e$ of sputtered silica measured by SEM (Figure 1b). Moreover, to obtain a computed structure as close as possible to real 25 sample, we needed to fill the voids between the two kinds of spheres of the model. For this, we added a rectangular layer of index $\mathrm{n}_{2}$ and of width $e$ between the centers of the two layers.
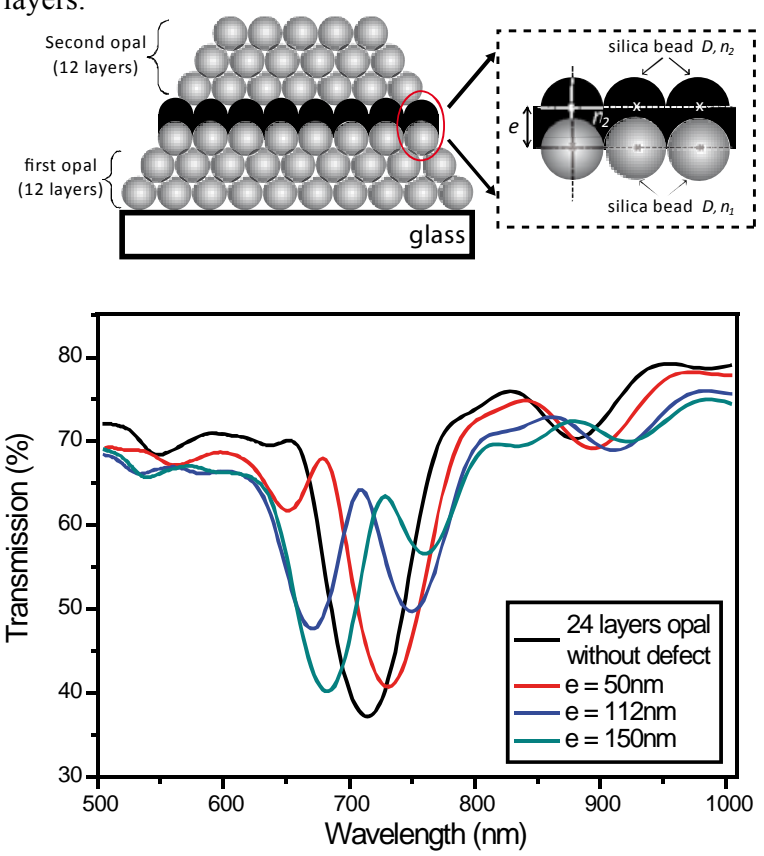

30 Figure 9: Top: Schematic of the computed structure. Down: $0^{\circ}-$ transmission spectra for an opal without defect (black line) and with defects of different thicknesses (red line: $50 \mathrm{~nm}$, blue line: 112 $\mathrm{nm}$, green line: $150 \mathrm{~nm}$ ).

On Figure 9 , we plotted the $0^{\circ}$-transmitted spectra for a 24 35 layers-opal without planar defect and for heterostructures with different values of the defect thickness $e$, ranging from $50 \mathrm{~nm}$ to $150 \mathrm{~nm}$. One can observe a broad dip of transmission, corresponding to the peak of reflection on experimental reflection spectra, which is the signature of the 40 photonic stopband. Inside this broad dip, a sharper peak (corresponding to an increase of the transmission) appears if a defect layer is embedded between the two opals. It corresponds to the passband created by the disruption of the opal periodicity due to the defect layer. For $e=112 \mathrm{~nm}$, the 45 defect layer thickness measured on the studied heterostructure, the defect mode is perfectly centered on the stopband as observed on reflection spectra for the real sample. By changing the value of $e$, its spectral position can be tuned inside de stopband.

\section{${ }_{50}$ Construction of the broad light source}

We consider a Gaussian source $S(\lambda)$ with a full width at half maximum $\delta \lambda$ centered on $\lambda_{\mathrm{c}}=690 \mathrm{~nm}$ which illuminates the sample and reconstruct the corresponding fluorescence intensity as:

55

$$
I_{\text {reconst }}(\theta)=\sum_{\lambda} I_{\text {meas }}(\lambda, \theta) \times \frac{S(\lambda)}{I_{\text {meas }}\left(\lambda, 60^{\circ}\right)}
$$

In this relationship, $\operatorname{Imeas}(\lambda, \theta)$ corresponds to the ${ }_{60}$ experimental emission diagrams, $\operatorname{Imeas}\left(\lambda, 60^{\circ}\right)$ to the experimental emission diagrams at $60^{\circ}$, far from the stopband of the opal (see Figure 4), and it is used for normalization by the $\mathrm{CdTeSe} / \mathrm{ZnS}$ nanocrystals intrinsic spectrum.

${ }_{65}$ The reconstructed signal is normalized by the same signal, $I_{\text {reconst }}(\theta)$, in the case of the reference sample (opal without planar defect).

\section{Acknowledgments}

The authors would like to thank their colleagues from INSP, ${ }_{70}$ Dominique Demaille for SEM measurements and Stéphane Chenot for silica sputtering.

The collaboration between INSP and IMS was supported by a Projet International de Coopération Scientifique (PICS 5724) between CNRS and VAST.

75 This work was supported by French state funds managed by the ANR within the Investissements d'Avenir programme under reference ANR-11-IDEX-0004-02, and more specifically within the framework of the Cluster of Excellence MATISSE and by the Direction Générale des 80 Armées (ANR-DGA "Calypso" and part of the financial support of Céline Bourdillon's PhD).

\section{Notes and references}

a Sorbonne Universités, UPMC Univ Paris 06, UMR 7588, INSP, ${ }_{85}$ F-75005, Paris, France

${ }^{b}$ CNRS, UMR 7588, Institut des NanoSciences de Paris, F-75005, Paris, France

schwob@insp.jussieu.fr

${ }^{c}$ Institute of Materials Science, Vietnam Academy of Science and

90 Technology, 18 Hoang Quoc Viet road, Cau Giay distr., Hanoi, Vietnam 
1 C. López, Adv. Mater., 2003, 15, 1679-1704.

2 M. Campbell, D. N. Sharp, M. T. Harrison, R. G. Denning and A. J. Turberfield, Nature, 2000, 404, 53-56.

3 G. Subramanian, V. N. Manoharan, J. D. Thorne and D. J. Pine, Adv. Mater., 1999, 11, 1261-1265.

4 B. T. Holland, C. F. Blanford and A. Stein, Science, 1998, 281, $10 \quad 538-540$.

5 P. Massé, S. Reculusa, K. Clays and S. Ravaine, Chem. Phys. Lett., 2006, 422, 251-255.

156 J.-F. Dechézelles, T. Aubert, F. Grasset, S. Cordier, C. Barthou, C. Schwob, A. Maître, R. A. L. Vallée, H. Cramail and S. Ravaine, Phys. Chem. Chem. Phys., 2010, 12, 11993 11999 .

207 P. Jiang, J. F. Bertone, K. S. Hwang and V. L. Colvin, Chem. Mater., 1999, 11, 2132-2140.

8 H. Cong and W. Cao, Langmuir, 2003, 19, 8177-8181.

259 P. V. Braun, S. A. Rinne and F. García-Santamaría, Adv Mater., 2006, 18, 2665-2678.

10 P. N. Hong, P. Bénalloul, Z. Guennouni-Assimi, R. Farha, C. Bourdillon, M.-C. Fauré, M. Goldmann, W. Marcillac, L.

30 Coolen, A. Maître and C. Schwob, Opt. Quantum Electron., 2014, 1-11.

11 P. N. Hong, P. Benalloul, L. Coolen, A. Maître and C. Schwob, J. Mater. Chem. C, 2013, 1, 5381-5386.

12 E. P. Petrov, V. N. Bogomolov, I. I. Kalosha and S. V. Gaponenko, Phys. Rev. Lett., 1998, 81, 77-80.

13 S. V. Gaponenko, V. N. Bogomolov, E. P. Petrov, A. M.

40 Kapitonov, D. A. Yarotsky, I. I. Kalosha, A. A. Eychmueller, A. L. Rogach, J. McGilp, U. Woggon and F. Gindele, J. Light. Technol., 1999, 17, 2128 .

14 L. Bechger, P. Lodahl and W. L. Vos, J. Phys. Chem. B, 2005, $45 \quad \mathbf{1 0 9}, 9980-9988$.

15 Z.-Y. Li and Y. Xia, Phys. Rev. A, 2001, 63, 043817.

16 M. Barth, R. Schuster, A. Gruber and F. Cichos, Phys. Rev. 50 Lett., 2006, 96, 243902.

17 B. H. Husken, A. F. Koenderink and W. L. Vos, J. Phys. Chem. C, 2012, 117, 3431-3439.

5518 C. Vion, C. Barthou, P. Bénalloul, C. Schwob, L. Coolen, A. Gruzintev, G. Emel'chenko, V. Masalov, J.-M. Frigerio and A Maître, J. Appl. Phys., 2009, 105, 113120.

19 M. D. Leistikow, A. P. Mosk, E. Yeganegi, S. R. Huisman, A. 60 Lagendijk and W. L. Vos, Phys. Rev. Lett., 2011, 107, 193903.

20 Z.-Y. Li and Z.-Q. Zhang, Phys. Rev. B, 2001, 63, 125106.

21 H. Frederich, F. Wen, J. Laverdant, L. Coolen, C. Schwob and A. Maître, Opt. Express, 2011, 19, 24424

22 H. Frederich, F. Wen, J. Laverdant, W. D. de Marcillac, C. Schwob, L. Coolen and A. Maître, Plasmonics, 2014, 9, $917-$ 924.

23 A. Hatef and M. R. Singh, Phys. Rev. A, 2010, 81, 063816.

24 W. Wang and S. A. Asher, J. Am. Chem. Soc., 2001, 123, $12528-12535$
25 C. Fenzl, T. Hirsch and O. S. Wolfbeis, Angew. Chem. Int. Ed., 2014, 53, 3318-3335.

26 W. Stöber, A. Fink and E. Bohn, J. Colloid Interface Sci., $80 \quad 1968,26,62-69$.

27 G. H. Bogush, M. A. Tracy and C. F. Zukoski IV, J. NonCryst. Solids, 1988, 104, 95-106.

${ }_{85} 28$ J. D. Joannopoulos, R. D. Meade and J. N. Winn, Photonic Crystals: Molding the Flow of Light, Princeton University Press, 1995.

29 A. Avoine, P. N. Hong, H. Frederich, J.-M. Frigerio, L.

90 Coolen, C. Schwob, P. T. Nga, B. Gallas and A. Maître, Phys. Rev. B, 2012, 86, 165432.

30 A. Taflove and S. C. Hagness, Computational Electrodynamics: The Finite-Difference Time-Domain Method. 95 Third edition, Norwood MA, Artech House., 2005. 